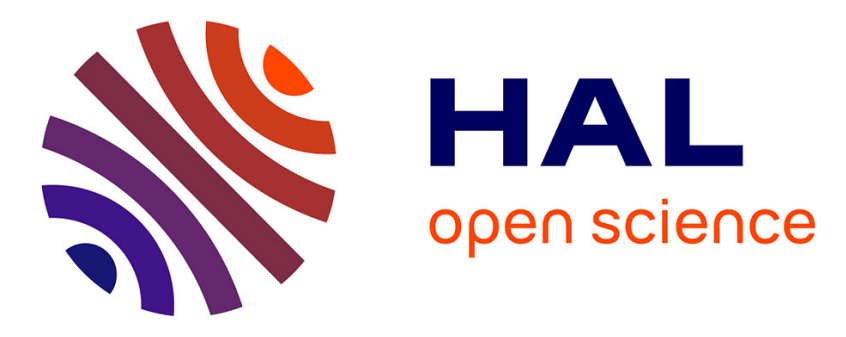

\title{
Real-time line projection for fast terahertz spectral computed tomography
}

Emmanuel Abraham, Yoshiyuki Ohgi, Masa-Aki Minami, Mukesh Jewariya, Masaya Nagai, Tsutomu Araki, Takeshi Yasui

\section{To cite this version:}

Emmanuel Abraham, Yoshiyuki Ohgi, Masa-Aki Minami, Mukesh Jewariya, Masaya Nagai, et al.. Real-time line projection for fast terahertz spectral computed tomography. Optics Letters, 2011, 36 (11), pp.2119-2121. 10.1364/OL.36.002119 . hal-00608296

\section{HAL Id: hal-00608296 https://hal.science/hal-00608296}

Submitted on 28 Aug 2018

HAL is a multi-disciplinary open access archive for the deposit and dissemination of scientific research documents, whether they are published or not. The documents may come from teaching and research institutions in France or abroad, or from public or private research centers.
L'archive ouverte pluridisciplinaire HAL, est destinée au dépôt et à la diffusion de documents scientifiques de niveau recherche, publiés ou non, émanant des établissements d'enseignement et de recherche français ou étrangers, des laboratoires publics ou privés.

\section{(1)(1) $\$(0)$}

Distributed under a Creative Commons Attribution - NonCommercial - ShareAlikel 4.0 


\title{
Real-time line projection for fast terahertz spectral computed tomography
}

\author{
Emmanuel Abraham, ${ }^{1}$ Yoshiyuki Ohgi, ${ }^{2}$ Masa-aki Minami, ${ }^{2}$ Mukesh Jewariya, ${ }^{3}$ \\ Masaya Nagai, ${ }^{2}$ Tsutomu Araki, ${ }^{2}$ and Takeshi Yasui ${ }^{2,4, *}$ \\ ${ }^{1}$ Laboratoire Ondes et Matière d'Aquitaine (LOMA), University of Bordeaux CNRS, UMR 5798, \\ 351 Cours de la Libération, 33405 Talence, France \\ ${ }^{2}$ Graduate School of Engineering Science, Osaka University, Toyonaka, Osaka 560 8531, Japan \\ ${ }^{3}$ Renovation Center of Instruments for Science Education and Technology, Osaka University, Toyonaka, Osaka 560 0043, Japan \\ ${ }^{4}$ Institute of Technology and Science, University of Tokushima, Tokushima 560 8531, Japan \\ ${ }^{*}$ Corresponding author: yasui@me.tokushima u.ac.jp
}

\begin{abstract}
We demonstrated fast terahertz spectral computed tomography by using real time line projection of a terahertz beam. Two types of cross sectional images of continuously rotating samples have been measured in only a few seconds. From temporal data, a peak to peak sinogram and cross sections have been reconstructed using a filtered backprojection algorithm. Using fast Fourier transform from temporal data, spectral cross sections of the sample have been obtained.

OCIS codes: $110.6795,110.6955,120.3620,320.7090$.
\end{abstract}

In the field of three-dimensional (3D) imaging, x-ray computed tomography (CT) is a ubiquitous technique, which provides cross-sectional images of an object by analyzing the radiation transmitted by the sample at different projection angles. Then a filtered backprojection (FBP) algorithm using a Radon inverse transform makes it possible to reconstruct the cross-sectional images [1]. However, X-ray CT cannot be easily applied to soft materials such as plastics, papers, or paintings because of low absorption of the radiation. In this case, terahertz $(\mathrm{THz})$ radiation can provide an alternative CT method due to its moderate penetration in dry, nonpolar, and soft materials, insensitivity to optical scattering, free-space propagation, and low photon energy. Furthermore, THz waves effectively provide spectral images of the test object in the $\mathrm{THz}$ range for material characterization. Therefore, $\mathrm{THz}$ CT has been developed to visualize 3D objects composed by opaque soft materials such as plastics, papers, composites, etc. [2-7].

Ferguson et $a \bar{l}$. demonstrated that cross-sectional images could be obtained by measuring the transmitted amplitude and phase of broadband THz pulses at multiple projection angles [2]. The reconstruction algorithm used for the visualization of the cross-sectional images usually consists of application of the FBP algorithm from the projection data. However, a strong limitation in practical use is the long acquisition time required by a pulsed THz system based on a time-domain spectrometer (TDS), since it uses point-by-point scanning to record a $\mathrm{THz}$ image. A simple way to decrease the acquisition time of the temporal waveform is to use a fast scanning mechanical time delay. Another effective way to realize rapid data acquisition is the use of an asynchronous optical sampling THz-TDS [8]. Nonmechanical time-delay scanning up to $250 \mathrm{~Hz}$ has been effectively applied to $\mathrm{THz}$ CT [6]. However, signal integration is usually required to get good dynamic range. An alternative method is the development of depth-resolving $\mathrm{THz}$ imaging with tomosynthesis, which is similar to CT except that the number of projections is much smaller [9]. In the above case, only five projections were used instead of generally 15 to 20 in $\mathrm{THz}$ CT. However, the efficiency of this system is mainly limited to thin and wide samples (50 sheets of Post-it notes). Furthermore, those methods were still based on point-by-point scanning measurements. If the pixel data could be measured in parallel simultaneously, the data acquisition time would be greatly reduced.

Recently, real-time two-dimensional spatiotemporal (2D-ST) imaging in the $\mathrm{THz}$ region has been achieved by a combination of noncollinear electro-optical time-tospace conversion [10] and line focusing of a THz beam, which has been effectively applied to $\mathrm{THz}$ reflective tomography [11] and spectral imaging of moving objects $[12,13]$. In this Letter, to significantly decrease the acquisition time of $\mathrm{THz} \mathrm{CT}$, we propose an experimental arrangement in which the sample is illuminated by a line-focused $\mathrm{THz}$ beam to achieve real-time line projection across the sample without scanning. 2D-ST lineprojection data of continuously rotating samples have been measured with a $100 \mathrm{~Hz}$ acquisition rate and used for spectral imaging of the sample cross section.

With a $1 \mathrm{kHz}$ femtosecond Ti:sapphire regenerative amplifier $(900 \mu \mathrm{J} /$ pulse, $150 \mathrm{fs}, 800 \mathrm{~nm})$, an intense $\mathrm{THz}$ pulse is generated using the tilted pulse front excitation technique in a $\mathrm{LiNbO}_{3}$ crystal [14]. The accessible spectral bandwidth of the THz pulse extends from 0 to $2 \mathrm{THz}$, limited by the $\mathrm{THz}$ absorption in the $\mathrm{LiNbO}_{3}$ crystal. The $\mathrm{THz}$ beam is line-focused onto the sample with a THz cylindrical lens (THz-CL1, $f^{\prime}=100 \mathrm{~mm}$, Tsurupica), resulting in a line of illumination (20 mm long) across the sample, as shown in Fig. 1. Then, the THz projection line was imaged onto a ZnTe crystal by a combination of a THz plano-convex lens (THz-L, $f^{\prime}=100 \mathrm{~mm}$, Tsurupica) and a THz cylindrical lens (THz-CL2, $f^{\prime}=100 \mathrm{~mm}$, Tsurupica). For detection, the THz pulse passing through the sample and the infrared probe light were noncollinearly incident on the ZnTe crystal for a time-to-space conversion of the pulsed $\mathrm{THz}$ electric field and line imaging of the sample along the $\mathrm{THz}$ focal line via $2 \mathrm{D}$ free-space electro-optical sampling. The spatial intensity 


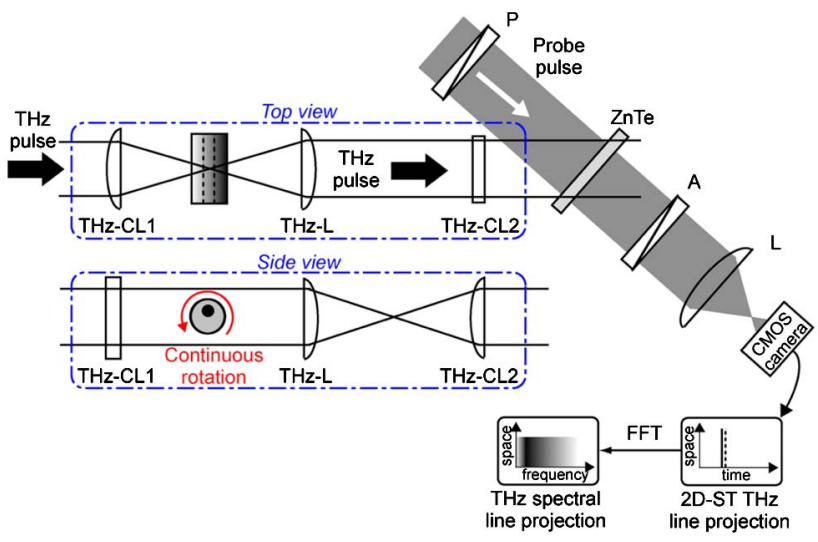

Fig. 1. (Color online) Experimental setup. THz CL1 and THz CL2, THz cylindrical lenses; THz L, THz plano convex lens; $P$, polarizer; $A$, analyzer; $L$, plano convex lens.

distribution of the probe beam was finally imaged with a plano-convex lens $\left(L, f^{\prime}=80 \mathrm{~mm}\right)$ onto a high-speed complementary metal-oxide semiconductor (CMOS) camera (Hamamatsu Photonic Intelligent Vision System, Hamamatsu, Japan, 12 bits, $232 \times 232$ pixels, 1000 frames/s) synchronized with the $1 \mathrm{kHz}$ laser pulse. A 2D-ST THz image was acquired at a frame rate of $500 \mathrm{~Hz}$ by the CMOS camera working in the dynamic subtraction mode [15], in which the temporal profile of the $\mathrm{THz}$ pulse (temporal range $=37 \mathrm{ps}$ ) and the $\mathrm{THz}$ lineimage of the projection (spatial range $=20 \mathrm{~mm}$ ) developed along the horizontal and vertical dimensions, respectively. Finally, owing to the $10 \mathrm{~ms}$ integration time of the CMOS camera, the 2D-ST image is acquired with a $100 \mathrm{~Hz}$ acquisition rate. In this case, the measured dynamic range is 945 , defined as the ratio of the signal amplitude (peak-to-peak) in the presence of the $\mathrm{THz}$ beam to that in the absence of the $\mathrm{THz}$ beam. The transverse resolution along the line projection attains the diffraction limit of the imaging system [12].

To perform fast THz CT, the sample was continuously rotated across the THz focal line as indicated in Fig. 1. The rotation time for a $360^{\circ}$ revolution of the sample was set to $6 \mathrm{~s}$. During this time, owing to the $10 \mathrm{~ms}$ continuous acquisition time of the $\mathrm{THz}$ line projection, up to 600 line-projection data were collected corresponding to an angular sampling of only $0.6^{\circ}$. This represents a large improvement for $\mathrm{THz}$ CT, where usually only 18 (angle step of $10^{\circ}$ ) or 36 (angle step of $5^{\circ}$ ) projections angles are selected owing to the long acquisition time of the experiment (point-by-point scanning measurement is more than $1 \mathrm{~h}$ ).

Finally, the combination of line projections and the FBP algorithm provide the sinogram and corresponding cross section of the sample at the tightly focusing position of the THz line. From the analysis of the temporal data, the peak-to-peak amplitude of the main $\mathrm{THz}$ pulse will provide information about global transmission of the sample in the $0-2 \mathrm{THz}$ spectral range. Using a fast Fourier transform (FFT) from the temporal data, spectral cross sections can be visualized at the spectral resolution of $27 \mathrm{GHz}$ in order to identify specific $\mathrm{THz}$ absorptions and perform $\mathrm{THz}$ spectral CT $[\underline{5}, \underline{6}]$.

To demonstrate the ability of fast cross-section reconstruction, we first investigated a simple sample consi-
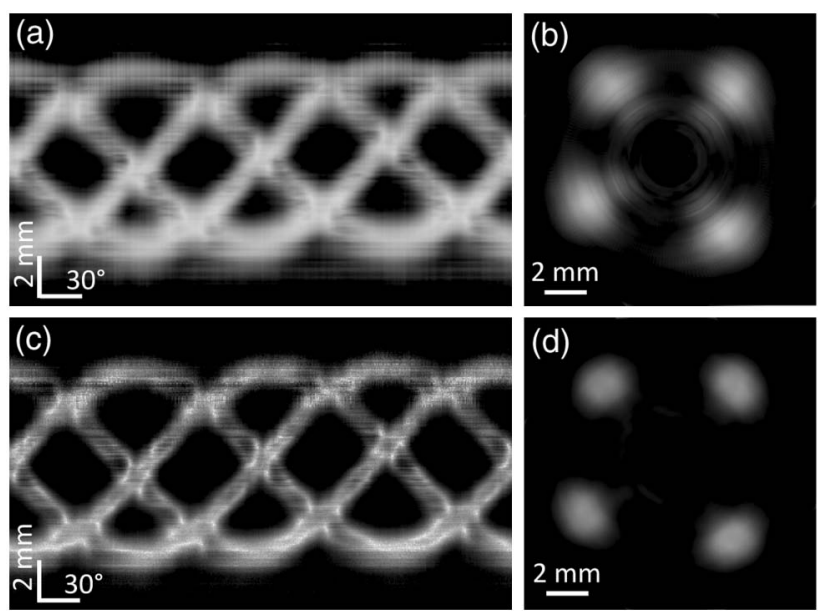

Fig. 2. Four metallic bars (2 mm diameter). (a) Peak to peak sinogram. (b) Peak to peak cross section. (c) Amplitude sino gram at $0.6 \mathrm{THz}$. (d) Amplitude cross section at $0.6 \mathrm{THz}$.

sting of four horizontal metallic bars (2 $\mathrm{mm}$ diameter) illuminated by the vertical $\mathrm{THz}$ line. As explained previously, up to 600 projection data were recorded, corresponding to the visualization of the sample through 600 different angles in only $6 \mathrm{~s}$, which represents a significant increase of the acquisition rate for THz CT. From these raw data, the first analysis consists in measuring the peak-to-peak amplitude of each $\mathrm{THz}$ waveform. Consequently, we can build the sinogram of the sample corresponding to the evolution of the $\mathrm{THz}$ line projection as a function of the rotation angle. Figure 2(a) shows the sinogram of the peak-to-peak amplitude for this sample, whose horizontal and vertical coordinates respectively give the rotation angle ( 600 points from 0 to $360^{\circ}$ ) and the THz projection-line image (232 points from 0 to $20 \mathrm{~mm}$ ). The white areas indicate a decrease in the peak-to-peak amplitude. Isolated distribution of the four metallic bars generates four overlapping sinusoidal waveforms in the sinogram with different original phases of the rotation angle. Using the standard FBP algorithm [1] , we reconstructed the cross-sectional image corresponding to the portion of the object illuminated by the $\mathrm{THz}$ line as shown in Fig. 2(b). Although some artifacts are visible (diffraction pattern around the metallic bars), the positions of the four metallic bars in the region corresponding to the illuminating $\mathrm{THz}$ vertical line are clearly identified, demonstrating the ability of the system to properly reconstruct in only $6 \mathrm{~s}$ cross-sectional images of simple samples.

Figures 2(c) and 2(d) illustrate the amplitude sinogram and cross section measured at $0.6 \mathrm{THz}$ after FFT of the temporal data (the white area indicates a decrease in the THz amplitude). Although no spectroscopic information can be obtained from this metallic sample, this sinogram and cross section exhibit a higher transverse resolution compared to temporal data owing to the shorter wavelength of the $\mathrm{THz}$ radiation $(500 \mu \mathrm{m})$. Also, the diffraction pattern around the four metallic bars has almost disappeared in the $0.6 \mathrm{THz}$ cross section. This indicates that, in the peak-to-peak cross section of Fig. 2(b), the diffraction pattern mainly comes from the interaction between the sample structure with a diameter of $2 \mathrm{~mm}$ 


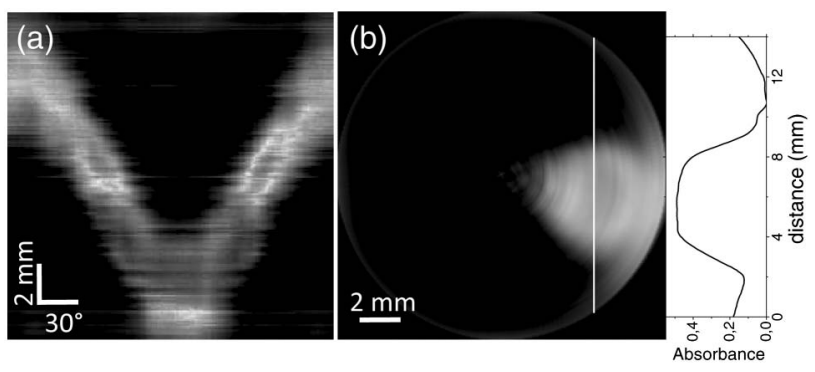

Fig. 3. Foam cylinder ( $16 \mathrm{~mm}$ diameter) with one off axis cylindrical hole (4 $\mathrm{mm}$ diameter) filled with lactose and polyethylene powder. (a) Amplitude sinogram at $0.53 \mathrm{THz}$. (b) Spectral cross section at $0.53 \mathrm{THz}$ and intensity profile along the vertical white line.

and the low frequency components of the $\mathrm{THz}$ radiation $(0-0.5 \mathrm{THz})$.

The second sample is a foam cylinder $(16 \mathrm{~mm}$ diameter) drilled with an off-axis cylindrical hole ( $4 \mathrm{~mm}$ diameter). To demonstrate $\mathrm{THz}$ spectral CT, the hole was filled with $\alpha$-lactose monohydrate sugar mixed with polyethylene powder (30\% lactose and $70 \%$ polyethylene). According to previous studies, lactose exhibits a $\mathrm{THz}$ spectral fingerprint at $0.53 \mathrm{THz}[13]$. With the same experimental conditions (rotation from 0 to $360^{\circ}$ in $6 \mathrm{~s}, 600$ projections, horizontal sample illuminated by the vertical THz line), Figs. 3(a) and 3(b) represent the amplitude sinogram measured at $0.5 \overline{\mathrm{THz}}$ and the corresponding reconstructed spectral cross section of the sample, respectively. First, the shape of the foam cylinder is not visible, owing to the low refractive index change from air to foam and the low absorption coefficient of the foam [Rohacell, (Evonik Industries, Germany) $\alpha=0.1 \mathrm{~cm}^{-1}$, refractive index $=1.15]$. In Figs. 3(a) and 3(b), the position and the size of the inner cylindrical hole is revealed due to the different transmission of the $\mathrm{THz}$ radiation in this part of the sample. The lower transmission is due to both refraction losses and lactose absorption at $0.53 \mathrm{THz}$. In the cross-section image, we can observe that the shape of the hole is not regular, perhaps due to diffraction artifacts. The vertical intensity profile measured along the white line drawn in Fig. 3(b) is also presented, in which the pixel values have been plotted in logarithmic scale. The data reflect the sample absorbance, classically defined as the logarithmic ratio between the transmitted THz signal measured out of the sample and the THz signal measured along the vertical line drawn in Fig. 3(b). In the region of the lactose cylinder, the absorbance at $0.53 \mathrm{THz}$ has been evaluated to 0.5 in agreement with lactose concentration and sample thickness. In the future, we plan to extend the spectral bandwidth of the $\mathrm{THz}$ emission to perform broadband spectral CT up to several THz.

In summary, we demonstrated the potential of realtime $\mathrm{THz}$ line projection for fast THz spectral CT. Owing to the $10 \mathrm{~ms}$ acquisition time of $2 \mathrm{D}-\mathrm{ST} \mathrm{THz}$ images, lineprojection data have been recorded in real time associated with the continuous rotation of the sample. With this system, by FFT of the time dimension in the 2D-ST THz image, fast THz spectral CT has been achieved at the acquisition time of $6 \mathrm{~s}$. Further developments include additional horizontal scanning of the sample associated with the continuous rotation to perform full 3D reconstruction of volumetric objects.

E. Abraham is grateful to the Invitation Fellowship Programs for Research, Japan Society for the Promotion of Science (JSPS), Japan. This work was supported by Grants-in-Aid for Scientific Research 21650111 from the Ministry of Education, Culture, Sports, Science \& Technology (MEXT), Japan. We also acknowledge the Renovation Center of Instruments for Science Education and Technology (Osaka University), and Tateishi Science and Technology Foundation for financial support.

\section{References}

1. G. T. Herman, Image Reconstruction from Projections The Fundamentals of Computerized Tomography (Academic, 1980).

2. B. Ferguson, S. Wang, D. Gray, D. Abbot, and X. C. Zhang, Opt. Lett. 27, 1312 (2002).

3. K. L. Nguyen, M. L. Johns, L. F. Gladden, C. H. Worral, P. Alexander, H. E. Beere, M. Pepper, D. A. Ritchie, J. Alton, S. Barbieri, and E. H. Linfield, Opt. Express 14, 2123 (2006).

4. E. Abraham, A. Younus, C. Aguerre, P. Desbarats, and P. Mounaix, Opt. Commun. 283, 2050 (2010).

5. A. Brahm, M. Kunz, S. Riehemann, G. Notni, and A. Tünnermann, Appl. Phys. B 100, 151 (2010).

6. E. Kato, S. Nishina, A. Irisawa, T. Yamashita, M. Imamura, and K. Kawase, in Proceedings of IEEE Conference on 35th International Conference on Infrared, Millimeter and Terahertz Waves (IEEE, 2010), paper Mo P.67.

7. B. Recur, A. Younus, S. Salort, P. Mounaix, B. Chassagne, P. Desbarats, J. P. Caumes, and E. Abraham, Opt. Express 19, 5105 (2011).

8. T. Yasui, E. Saneyoshi, and T. Araki, Appl. Phys. Lett. 87, 061101 (2005).

9. N. Sunaguchi, Y. Sasaki, N. Maikusa, M. Kawai, T. Yuasa, and C. Otani, Opt. Express 17, 9558 (2009).

10. J. Shan, A. S. Weling, E. Knoesel, L. Bartels, M. Bonn, A. Nahata, G. A. Reider, and T. F. Heinz, Opt. Lett. 25, 426 (2000).

11. T. Yasuda, T. Yasui, T. Araki, and E. Abraham, Opt. Commun. 267, 128 (2006).

12. T. Yasui, K. Sawanaka, A. Ihara, E. Abraham, M. Hashimoto, and T. Araki, Opt. Express 16, 1208 (2008).

13. M. Schirmer, M. Fujio, M. Minami, J. Miura, T. Araki, and T. Yasui, Biomed. Opt. Express 1, 354 (2010).

14. J. Jewariya, M. Nagai, and K. Tanaka, J. Opt. Soc. Am. B 26, A101 (2009).

15. F. Miyamaru, T. Yonera, M. Tani, and M. Hangyo, Jpn. J. Appl. Phys. 43, L489 (2004). 